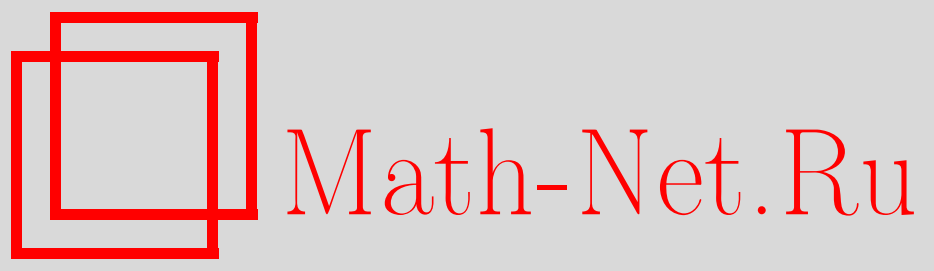

В. М. Журавлев, Принцип суперпозиции и точные решения уравнения нелинейной диффузии, ТМФ, 2015, том 183, номер 1, 36-50

DOI: https://doi.org/10.4213/tmf8781

Использование Общероссийского математического портала Math-Net.Ru подразумевает, что вы прочитали и согласны с пользовательским соглашением http://www . mathnet.ru/rus/agreement

Параметры загрузки:

IP : 54.89 .56 .158

26 апреля 2023 г., 12:53:27

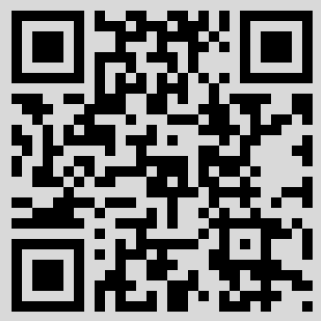




\title{
ПРИНЦИП СУПЕРПОЗИЦИИ И ТОЧНЫЕ РЕШЕНИЯ УРАВНЕНИЯ НЕЛИНЕЙНОЙ ДИФФУЗИИ
}

\begin{abstract}
Излагается метод построения точных решений уравнений нелинейной диффузии в одномерном координатном пространстве на основе использования специального принципа суперпозиции. В качестве уравнения нелинейной диффузии рассматриваются уравнения вида $n_{t}-(\ln n)_{x x}+\mu n+\gamma n^{2}-g=0$, играющие важную роль в задаче о формировании регулярных структур в нелинейных средах под действием внешних источников излучения. Метод основан на использовании дифференциальных свойств полиномов от функциональных параметров. Приводятся конкретные решения и анализируются некоторые их общие свойства.
\end{abstract}

Ключевые слова: уравнения нелинейной диффузии, точные решения, принцип суперпозиции, регулярные структуры.

DOI: $10.4213 / \operatorname{tmf} 8781$

\section{1. ВВЕДЕНИЕ}

Формирование регулярных структур в различных веществах под действием внешних потоков излучения является одной из актуальных задач современной теории конденсированных сред. Один из способов объяснения возникающих регулярных структур опирается на предположение, что основную роль в их формировании играет нелинейная диффузия, т. е. зависимость коэффициента диффузии от концентрации диффундирующей примеси. В данном подходе функциональная зависимость коэффициента диффузии предполагается такой, что увеличение концентрации дефектов уменьшает скорость их диффузии. Это и приводит к формированию больших концентраций примеси. Такого рода модели исследовались ранее в связи с задачами распространения тепла в средах с нелинейными коэффициентами теплопроводности [1], [2].

Для появления регулярных структур в этом случае необходимо также наличие каких-либо законов сохранения или симметрий в среде. С математической точки

*Научно-исследовательский технологический институт им. С. П. Капицы, Ульяновский государственный университет, Ульяновск, Россия. E-mail: zhvictorm@gmail.com 
зрения особыми свойствами обладает коэффициент диффузии вида

$$
D=\frac{\mathcal{D}_{0}}{n+n_{\mathrm{s}}}
$$

где $\mathcal{D}_{0}$ и $n_{\mathrm{s}}$ - некоторые функции времени, $n$ - концентрация изучаемых дефектов, изменяющаяся в пространстве и времени. Этот коэффициент диффузии отвечает требованию уменьшения скорости диффузии при увеличении концентрации, с другой стороны, уравнения диффузии с таким коэффициентом обладают специфическими симметрийными свойствами. Уравнения теплопроводности или диффузии с таким коэффициентом диффузии исследовались, например, в работах [3]-[5]. В этих работах было показано, что существуют обширный класс точных решений и специфический нелинейный принцип суперпозиции [5]-[7]. Поэтому при построении моделей процессов, приводящих к образованию регулярных структур в твердых телах, модели с коэффициентом диффузии (1) могут представлять особый интерес.

В работах [3]-[5] исследования проводились для уравнений диффузии или теплопроводности с коэффициентом (1) в двумерном координатном пространстве. Однако для ряда прикладных задач необходимо построение точных решений такого типа уравнений в одномерном и трехмерном координатных пространствах. В связи с этим в настоящей работе рассматривается задача нахождения точных решений уравнений нелинейной диффузии с коэффициентом (1) в случае одномерного координатного пространства, но с нелинейным источником в правой части в форме квадратичной функции концентрации. По аналогии с работой [5] для построения точных решений применяется нелинейный принцип суперпозиции в расширенной по сравнению со статьей [5] форме. Это позволяет применить метод и в случае изменяющихся со временем коэффициентов нелинейного источника.

\section{2. УРАВНЕНИЕ НЕЛИНЕЙНОЙ ДИФФУЗИИ}

Используя коэффициент диффузии (1), рассмотрим одномерное уравнение нелинейной диффузии в следующем виде:

$$
\frac{\partial n_{0}}{\partial t}=\frac{\partial}{\partial x}\left(\frac{\mathcal{D}_{0}}{n_{0}+n_{\mathrm{s}}} \frac{\partial n_{0}}{\partial x}\right)-p(t) n_{0}-r(t) n_{0}^{2}+I(t) .
$$

Здесь $n_{0}(x, t)$ - концентрация диффундирующей примеси или дефектов, $t$ - время, $x$ - координата, вдоль которой происходит диффузия. Источник в правой части этого уравнения описывает процессы возникновения и поглощения примеси или дефектов в зависимости от их локальной концентрации. Квадратичное по $n_{0}$ слагаемое в этом источнике обычно связывают с саморекомбинацией дефектов или примеси, а слагаемое с первой степенью $n_{0}$ отвечает за "диссипацию" за счет, например, самораспада или ухода за границы среды. Коэффициенты $p(t)$ и $r(t)$ этого уравнения будем в общем случае полагать функциями времени $t$. Функция $I(t)$ в правой части описывает внешний источник примеси или дефектов, например, за счет равномерного облучения среды. Такой тип источника является общим для многих задач формирования волн и структур в нелинейных средах с диффузией. 
Вводя функцию $n=n_{0}+n_{\mathrm{s}}$ и полагая $n_{\mathrm{s}}=n_{\mathrm{s}}(t)$, преобразуем уравнение $(2)$ к виду

$$
\frac{\partial n}{\partial t}=\frac{\partial}{\partial x}\left(\frac{\mathcal{D}_{0}}{n} \frac{\partial n}{\partial x}\right)-\mu(t) n-\gamma(t) n^{2}+g(t)
$$

Здесь

$$
\mu(t)=p(t)-2 \gamma(t) n_{\mathrm{s}}, \quad g(t)=I(t)+\dot{n}_{\mathrm{s}}+p(t) n_{\mathrm{s}}-\gamma(t) n_{\mathrm{s}}^{2}, \quad \gamma(t)=r(t) .
$$

На первом этапе построения простых точных решений мы ограничимся случаем $g=0$. Это ограничение связано с тем, что при этом условии существуют простейшие решения. На основе этих решений в настоящей работе построены более сложные суперпозиционные решения, с помощью которых рассмотрен вопрос о существовании решений с $g(t) \neq 0$ в правой части.

\section{3. ПРОСТЫЕ ТОЧНЫЕ РЕШЕНИЯ ПРИ $g=0$}

Наличие простых решений для уравнения нелинейной диффузии в двумерном координатном пространстве было показано в работе [5]. Однако в отличие от работы [5] здесь мы рассмотрим одномерный вариант уравнения нелинейной диффузии, но с более сложным нелинейным источником, содержащим квадратичную нелинейность с переменными во времени коэффициентами.

По аналогии с работой [5] введем вспомогательную функцию $\Phi=1 / n$, в результате чего уравнение (3) запишется как

$$
\frac{\partial}{\partial t} \frac{1}{\Phi}+\mathcal{D}_{0} \frac{\partial^{2} \ln \Phi}{\partial x^{2}}+\frac{\mu(t)}{\Phi}+\frac{\gamma(t)}{\Phi^{2}}=0 .
$$

Его можно привести к виду

$$
-\Phi_{t}+\mathcal{D}_{0}\left(\Phi \Phi_{x x}-\left(\Phi_{x}\right)^{2}\right)+\mu \Phi+\gamma=0
$$

Будем искать решение этого уравнения в виде

$$
\Phi=A(t)+B(t) e^{k x}+C(t) e^{-k x}
$$

где $A(t), B(t), C(t)$ - функции времени, для которых необходимо получить уравнения и решить их, а $k$ - произвольный, вообще говоря, комплексный, параметр. Подставляя (6) в (5), находим уравнения для функций $A, B, C$ :

$$
\begin{aligned}
& \dot{A}=4 k^{2} \mathcal{D}_{0} B C+\mu(t) A+\gamma(t), \\
& \dot{B}=4 k^{2} \mathcal{D}_{0} A B+\mu(t) B, \\
& \dot{C}=4 k^{2} \mathcal{D}_{0} A C+\mu(t) C .
\end{aligned}
$$

Введем обозначение $\dot{\chi}=4 k^{2} \mathcal{D}_{0} A+\mu$. Тогда уравнение для $\chi$ можно записать в такой форме:

$$
\ddot{\chi}=16 k^{4} \mathcal{D}_{0}^{2} B_{0} C_{0} e^{2 \chi}+(\dot{\chi}-\mu(t)) \mu(t)+\gamma(t) 4 k^{2} D_{1}+\dot{\mu} .
$$


Решения уравнений для $A, B$ и $C$ имеют вид

$$
A=\frac{1}{4 k^{2} \mathcal{D}_{0}}(\dot{\chi}-\mu(t)), \quad B=B_{0} e^{\chi}, \quad C=C_{0} e^{\chi}
$$

Сделаем преобразование $\chi(t)=\Theta(t)+(1 / 2) \ln \left|B_{0} C_{0}\right|$. Тогда для функции $\Theta$ уравнение примет вид

$$
\ddot{\Theta}=16 k^{4} \mathcal{D}_{0}^{2} \varepsilon e^{2 \Theta}+(\dot{\Theta}-\mu) \mu+\gamma 4 k^{2} \mathcal{D}_{0}+\dot{\mu},
$$

где $\varepsilon=\operatorname{sgn}\left(B_{0} C_{0}\right)$; тогда можно записать

$$
A=\frac{1}{4 k^{2} \mathcal{D}_{0}}(\dot{\Theta}-\mu), \quad B=\varepsilon_{1} Q_{0} e^{\Theta}, \quad C=\varepsilon_{2} \frac{1}{Q_{0}} e^{\Theta},
$$

где

$$
Q_{0}=\sqrt{\frac{\left|B_{0}\right|}{\left|C_{0}\right|}}, \quad \varepsilon_{1}=\operatorname{sgn} B_{0}, \quad \varepsilon_{2}=\operatorname{sgn} C_{0} .
$$

Кроме решений вида $(6)$, существуют и решения $\Phi(x, t)$, квадратичные по координате $x$. Эти решения можно представить в виде

$$
\Phi(x, t)=A_{1}(t)+B_{1}(t) x+C_{1}(t) x^{2} .
$$

Уравнения для коэффициентов $A_{1}, B_{1}, C_{1}$ в этом случае записываются как

$$
\begin{aligned}
& \dot{A}_{1}=2 \mathcal{D}_{0} C_{1} A_{1}+\mu(t) A-\mathcal{D}_{0} B_{1}^{2}+\gamma(t), \\
& \dot{B}_{1}=\left(-2 \mathcal{D}_{0} C_{1}+\mu(t)\right) B_{1}, \\
& \dot{C}_{1}=\left(-2 \mathcal{D}_{0} C_{1}+\mu(t)\right) C_{1} .
\end{aligned}
$$

Решение двух последних уравнений таково:

$$
B_{1}=\frac{B_{0}}{\chi(t)} \exp \left\{\int \mu(t) d t\right\}, \quad C_{1}=\frac{1}{2 \mathcal{D}_{0}} \frac{d \ln \chi(t)}{d t},
$$

где

$$
\chi(t)=\chi_{1} \int^{t} \exp \left\{\int^{t^{\prime}} \mu\left(t^{\prime \prime}\right) d t^{\prime \prime}\right\} d t^{\prime}+\chi_{0},
$$

a $\chi_{0}, \chi_{1}-$ постоянные интегрирования. Линейное уравнение относительно $A(t)$ peшается при этом без труда.

Таким образом, уравнения (4) и (5) имеют точные решения

$$
\begin{aligned}
& \Phi=\frac{1}{4 k^{2} \mathcal{D}_{0}}(\dot{\Theta}-\mu(t))+A_{0} e^{\Theta+k x}+\varepsilon \frac{1}{A_{0}} e^{\Theta-k x}, \\
& \Phi=A_{1}(t)+B_{1}(t) x+C_{1}(t) x^{2},
\end{aligned}
$$

где $k, \mathcal{D}_{0}$ - произвольные постоянные, $\varepsilon=\{ \pm 1,0\}, A_{0}=\varepsilon_{1} Q_{0}$, при условии, что $\Theta(t)$ является решением уравнения (8), а функции $A_{1}, B_{1}, C_{1}$ - решения уравнений (10). 
Соответствующие решения уравнения (2) имеют вид

$$
n=\frac{4 k^{2} \mathcal{D}_{0}}{(\dot{\Theta}-\mu(t))+\varepsilon_{1} 4 k^{2} \mathcal{D}_{0} e^{\Theta(t)}\left(e^{k\left(x-x_{0}\right)}+\varepsilon e^{-k\left(x-x_{0}\right)}\right)},
$$

где $x_{0}=-\ln \left|A_{0}\right|$, и

$$
n=\frac{1}{A_{1}(t)+B_{1}(t) x+C_{1}(t) x^{2}} ;
$$

при вещественных значениях $k$ функция $n$ удовлетворяет граничному условию $n \rightarrow 0$ при $|x| \rightarrow \infty$. В случае чисто мнимых значений $k=i q$ и условии $C_{0}=B_{0}^{*}$ решения для $\Phi$ и $n$ будут вещественными периодическими решениями по $x$ :

$$
\begin{aligned}
& \Phi=-\frac{1}{4 q^{2} \mathcal{D}_{0}}(\dot{\Theta}-\mu)+\left|B_{0}\right| e^{\Theta} \cos (q x+\varphi), \\
& n=\frac{4 q^{2} \mathcal{D}_{0}}{(\mu(t)-\dot{\Theta})+4 q^{2} \mathcal{D}_{0}\left|B_{0}\right| e^{\Theta} \cos (q x+\varphi)},
\end{aligned}
$$

где $\varphi=\arg B_{0}$.

Отметим, что в случае $C_{0}=0$ (или $B_{0}=0$ ) последнее уравнение становится линейным

$$
\ddot{\Theta}=(\dot{\Theta}-\mu(t)) \mu(t)+\gamma(t) 4 k^{2} \mathcal{D}_{0}+\dot{\mu} .
$$

В этом случае решение для $\Phi$ будет иметь такой вид:

$$
\Phi=\frac{1}{4 k^{2} \mathcal{D}_{0}}(\dot{\Theta}-\mu)+B_{0} e^{\Theta+k x} .
$$

Для полиномиальных решений имеются две аналогичные редукции. При $\chi_{1}=0$ в $(11)$ имеем $C_{1}(t) \equiv 0$, и соответствующим образом упрощаются уравнения для $A_{1}(t), B_{1}(t)$. При этом

$$
\Phi=A_{1}(t)+B_{0} e^{\Omega(t)} x, \quad \Omega(t)=\int_{0}^{t} \mu\left(t^{\prime}\right) d t^{\prime} .
$$

При $B_{0}=0$ имеем $B(t) \equiv 0$ и $\Phi=A_{1}(t)+C_{1}(t) x^{2}$.

\section{4. НЕЛИНЕЙНЫЙ ПРИНЦИП СУПЕРПОЗИЦИИ}

Для построения более общих решений уравнения (5) и уравнения (4) с $g=0$ воспользуемся методом, предложенным в работе [5], который опирается на свойство уравнения нелинейной диффузии - специальный принцип суперпозиции.

Для формулировки принципа суперпозиции введем некоторые обозначения. Обозначим через $z_{k}, k=1, \ldots, N$, корни полинома

$$
U_{N}(z)=z^{N}+\sum_{k=0}^{N-1} p_{k} z^{k}=\prod_{j=1}^{N}\left(z-z_{j}\right) .
$$

Для сокращения записи также введем обозначение $\mathbf{z}=\left\{z_{1}, z_{2}, \ldots, z_{N}\right\}$. Согласно теореме Виета коэффициенты $p_{k}$ полинома $U_{N}(z)$ являются функциями его корней, 
это мы будем записывать как $p_{k}=(-1)^{N-k} P_{k}(\mathbf{z})$, где

$$
P_{0}(\mathbf{z})=\prod_{j=1}^{N} z_{j}, \quad P_{1}(\mathbf{z})=\sum_{i=1}^{N} \prod_{\substack{1 \leqslant j \leqslant N, j \neq i}} z_{j}, \quad \ldots \quad, \quad P_{N-1}(\mathbf{z})=\sum_{i=1}^{N} z_{i}, \quad P_{N}=1 .
$$

Обратим внимание на существование тождеств

$$
\begin{aligned}
& P_{k}\left(z-z_{1}, \ldots, z-z_{N}\right)=\frac{1}{k !} \frac{\partial^{k}}{\partial z^{k}} P_{0}\left(z-z_{1}, \ldots, z-z_{N}\right), \\
& p_{k}=\left.P_{k}\left(z-z_{1}, \ldots, z-z_{N}\right)\right|_{z=0}, \quad k=1, \ldots, N-1 .
\end{aligned}
$$

Рассмотрим совокупности решений (14) и (15)

$$
\begin{aligned}
\Phi_{i} & =\frac{1}{4 k^{2} \mathcal{D}_{0}}(\dot{\Theta}-\mu)+B_{i} e^{\Theta+k x}, \\
\Phi_{i} & =A(t)+B_{i} x e^{\Omega(t)}, \quad i=1, \ldots, N,
\end{aligned}
$$

отличающихся лишь числовыми значениями параметров $B_{i}$. Для унификации вычислений функция $A_{1}(t)$ в решениях типа (15) обозначена как $A(t)$. Введем обозначения для решений (16)

$$
Z(x, t)=A(t) e^{-\Theta} e^{-k x}, \quad z_{i}=-B_{i}
$$

и обозначения для решений (17)

$$
Z(x, t)=\frac{A(t)}{x} e^{-\Omega}, \quad z_{i}=-B_{i} .
$$

Тогда в обоих случаях

$$
\Phi_{i}=\frac{A}{Z}\left(Z-z_{i}\right), \quad i=1, \ldots, N
$$

Для сокращения записи положим $\mathbf{Z}=\left\{Z-z_{1}, \ldots, Z-z_{N}\right\}$. Тогда имеют место следующие тождества:

$$
\begin{aligned}
& P_{j}\left(\Phi_{1}, \ldots, \Phi_{N}\right)=\left(\frac{A}{Z}\right)^{N-j} P_{j}(\mathbf{Z}), \\
& P_{j}\left(\Phi_{1}, \ldots, \Phi_{N}\right)=\left(\frac{A}{Z}\right)^{N-j} \frac{1}{j !} \frac{\partial^{j}}{\partial Z^{j}} P_{0}(\mathbf{Z}), \quad j=0, \ldots, N-1 .
\end{aligned}
$$

Производные по $x$ и $Z(x, t)$ для любой функции $F(Z)$ связаны следующими простыми соотношениями:

$$
\begin{aligned}
\frac{\partial}{\partial x} F(Z) & =\frac{\partial Z}{\partial x} \frac{\partial}{\partial Z} F(Z)=-k Z \frac{\partial}{\partial Z} F(Z), \\
\frac{\partial}{\partial x} F(Z) & =\frac{\partial Z}{\partial x} \frac{\partial}{\partial Z} F(Z)=-\frac{Z^{2}}{A} \frac{\partial}{\partial Z} F(Z) .
\end{aligned}
$$


Поскольку дальнейшие построения аналогичны как для решений (16), так и для решений (17), будем проводить вычисления только для случая (16). Решения типа (17) могут быть использованы аналогичным образом.

Используя соотношения для полиномов $P_{k}(\mathbf{Z})$, можно записать равенства

$$
\begin{aligned}
u_{N} & =\sum_{k=1}^{N} \frac{1}{\Phi_{k}}=\frac{P_{1}\left(\Phi_{1}(x, t), \ldots, \Phi_{N}(x, t)\right)}{P_{0}\left(\Phi_{1}(x, t), \ldots, \Phi_{N}(x, t)\right)}=\frac{Z}{A} \frac{P_{1}(\mathbf{Z})}{P_{0}(\mathbf{Z})}= \\
& =\frac{Z}{A} \frac{\partial}{\partial Z} \ln P_{0}(\mathbf{Z})=-\frac{1}{A k} \frac{\partial}{\partial x} \ln P_{0}(\mathbf{Z}) \\
\sum_{i=1}^{N} \frac{1}{\Phi_{i}^{2}} & =\frac{P_{1}^{2}\left(\Phi_{1}, \ldots, \Phi_{N}\right)}{P_{0}^{2}\left(\Phi_{1}, \ldots, \Phi_{N}\right)}-2 \frac{P_{2}\left(\Phi_{1}, \ldots, \Phi_{N}\right)}{P_{0}\left(\Phi_{1}, \ldots, \Phi_{N}\right)}= \\
& =-\frac{Z^{2}}{A^{2}} \frac{\partial^{2} \ln P_{0}(\mathbf{Z})}{\partial Z^{2}}=-\frac{1}{A}\left(Z \frac{\partial u_{N}}{\partial Z}-u_{N}\right)=\frac{1}{A k} \frac{\partial u_{N}}{\partial x}+\frac{1}{A} u_{N} .
\end{aligned}
$$

УТВЕРЖДЕНИЕ 1. Пусть $\Phi_{i}(x, t), i=1, \ldots, N,-$ совокупность точных решений уравнения (5) вида (16). Тогда функиия $u_{N}$, определенная соотношениями (21), при произвольных функциях $\mathcal{D}_{0}(t), \mu(t)$ u $\gamma(t)$, а также при произвольных значениях параметров $z_{i}=-B_{i}, k$ и произвольном $N$ удовлетворяет уравнению первого порядка

$$
\frac{\partial u_{N}}{\partial t}-\left(k A \mathcal{D}_{0}-\frac{\gamma}{A k}\right) \frac{\partial u_{N}}{\partial x}+\left(\mu+\frac{\gamma}{A}\right) u_{N}=0
$$

где функиия $A(t)$ определена соотношением (9).

ДокАЗАТЕЛЬСтво. Пусть $\Phi_{i}-$ совокупность решений (16), т. е. выполнены тождества

$$
\frac{\partial}{\partial t} \frac{1}{\Phi_{i}}+\mathcal{D}_{0} \frac{\partial^{2} \ln \Phi_{i}}{\partial x^{2}}+\frac{\mu(t)}{\Phi_{i}}+\frac{\gamma(t)}{\Phi_{i}^{2}}=0, \quad i=1, \ldots, N .
$$

Суммируя все эти тождества, получаем, что

$$
\frac{\partial}{\partial t} \sum_{i=1}^{N} \frac{1}{\Phi_{i}}+\mathcal{D}_{0} \frac{\partial^{2}}{\partial x^{2}} \ln \prod_{i=1}^{N} \Phi_{i}+\mu(t) \sum_{i=1}^{N} \frac{1}{\Phi_{i}}+\gamma(t) \sum_{i=1}^{N} \frac{1}{\Phi_{i}^{2}}=0 .
$$

Используя определение функции $u_{N}$ из (21), последнее уравнение приводим к следующему виду:

$$
\frac{\partial u_{N}}{\partial t}+\mathcal{D}_{0} \frac{\partial^{2}}{\partial x^{2}} \ln P_{0}\left(\Phi_{1}, \ldots, \Phi_{N}\right)+\mu(t) u_{N}+\gamma(t) \sum_{i=1}^{N} \frac{1}{\Phi_{i}^{2}}=0
$$

Воспользуемся теперь равенствами (20) и вторым тождеством из (21). Тогда уравнение (23) можно записать как

$$
\frac{\partial u_{N}}{\partial t}+\mathcal{D}_{0} k^{2} A Z \frac{\partial}{\partial Z}\left(\frac{Z}{A} \frac{\partial}{\partial Z} \ln P_{0}(\mathbf{Z})\right)+\mu(t) u_{N}+\frac{\gamma(t)}{A}\left(\frac{1}{k} \frac{\partial u_{N}}{\partial x}+u_{N}\right)=0 .
$$

Возвращаясь теперь к производным по $x$ с помощью (20), приходим окончательно к уравнению (22). Утверждение доказано. 
Доказанное утверждение указывает на то, что решения самого́ исходного уравнения (4), имеющие вид суперпозиционного решения $\Phi_{\mathrm{S}}$, можно построить, если специальным образом вычислять числа $B_{i}$.

\section{5. ОБОБЩЕННЫЕ ПОЛИНОМИАЛЬНЫЕ РЕШЕНИЯ}

Исследуем теперь вопрос о выводе уравнений, которым удовлетворяют суперпозиции решений (12) общего вида. Пусть $\Phi_{i}$ - совокупность решений вида (12), которые мы запишем как

$$
\Psi_{i}(x, t)=B(t)\left(T(t)+\tau_{i}(x)\right), \quad i=1, \ldots, N .
$$

Здесь

$$
\begin{gathered}
B(t)=e^{\Theta(t)}, \quad T(t)=\frac{1}{4 \mathcal{D}_{0} k^{2}}(\dot{\Theta}-\mu) e^{-\Theta}, \\
\tau_{i}(x)=B_{i} e^{-k x}+\varepsilon_{i} \frac{1}{B_{i}} e^{k x}, \quad \varepsilon_{i}= \pm 1 .
\end{gathered}
$$

Аналогичным образом можно исследовать и полиномиальные решения общего вида (13). Однако мы найдем только решения (12). Из определений (25) следует, что

$$
\frac{\partial}{\partial t} F(T)=\frac{\partial T}{\partial t} \frac{\partial}{\partial T} F(T)=\dot{T} \frac{\partial}{\partial T} F(T) .
$$

Для полиномов по $T$ выполняются свойства, аналогичные (18) и (19):

$$
\begin{aligned}
& P_{j}\left(\Psi_{1}, \ldots, \Psi_{N}\right)=B^{N-j}(t) P_{j}(\mathbf{T}), \\
& P_{j}\left(\Psi_{1}, \ldots, \Psi_{N}\right)=B^{N-j} \frac{1}{j !} \frac{\partial^{j}}{\partial T^{j}} P_{0}(\mathbf{T}), \quad j=0, \ldots, N-1,
\end{aligned}
$$

где введено обозначение $\mathbf{T}=\left\{T-\tau_{1}, \ldots, T-\tau_{N}\right\}$.

По аналогии с предыдущим анализом рассмотрим систему тождеств

$$
\begin{aligned}
v_{N} & =\sum_{i=1}^{N} \frac{1}{\Psi_{i}}=\frac{P_{1}\left(\Psi_{1}, \ldots, \Psi_{N}\right)}{P_{0}\left(\Psi_{1}, \ldots, \Psi_{N}\right)}= \\
& =\frac{1}{B} \frac{\partial \ln P_{0}(\mathbf{T})}{\partial T}=\frac{1}{B \dot{T}} \frac{\partial}{\partial t} \ln P_{0}(\mathbf{T}) \\
\sum_{i=1}^{N} \frac{1}{\Psi_{i}^{2}} & =\frac{P_{1}^{2}\left(\Psi_{1}, \ldots, \Psi_{N}\right)}{P_{0}^{2}\left(\Psi_{1}, \ldots, \Psi_{N}\right)}-2 \frac{P_{2}\left(\Psi_{1}, \ldots, \Psi_{N}\right)}{P_{0}\left(\Psi_{1}, \ldots, \Psi_{N}\right)}= \\
& =-\frac{1}{B^{2}} \frac{\partial^{2}}{\partial T^{2}} \ln P_{0}(\mathbf{T})=-\frac{1}{B \dot{T}} \frac{\partial v_{N}}{\partial t}-\frac{\dot{B}}{\dot{T} B^{2}} v_{N} .
\end{aligned}
$$

УТВЕРЖДЕНИЕ 2. Пусть $\Phi_{i}(x, t), i=1, \ldots, N,-$ совокупность точных решений уравнения (5) вида (24). Тогда функиия $v_{N}$, определенная соотношениями (26), при произвольных функциях $\mu(t)$ u $\gamma(t)$, а также при произволъных значениях параметров $B_{i}$ и $k$ и произвольном $N$ удовлетворяет уравнению второго порядка

$$
\frac{\partial}{\partial t}\left[\left(1-\frac{\gamma}{B \dot{T}}\right) \frac{\partial v_{N}}{\partial t}\right]+\mathcal{D}_{0} B \dot{T} \frac{\partial^{2} v_{N}}{\partial x^{2}}+\frac{\partial}{\partial t}\left[\left(\mu-\frac{\gamma \dot{B}}{\dot{T} B^{2}}\right) v_{N}\right]=0,
$$

где функиии $B(t)$ и $T(t)$ определены соотношениями (25). 
ДоКАЗАТЕЛЬСТво строится аналогично доказательству утверждения 1. Используя соотношения (26), как и при доказательстве утверждения 1 , получаем уравнение

$$
\left(1-\frac{\gamma}{B \dot{T}}\right) \frac{\partial v_{N}}{\partial t}+\mathcal{D}_{0} \frac{\partial^{2}}{\partial x^{2}} \ln P_{0}(\mathbf{T})+\left(\mu-\frac{\gamma \dot{B}}{\dot{T} B^{2}}\right) v_{N}=0 .
$$

Дифференцируя его по $t$, приходим к неавтономному уравнению второго порядка (22), содержащему в качестве неизвестной только $v_{N}$, что и доказывает утверждение.

Полученный результат интересен с точки зрения его использования для построения решений уравнений телеграфного типа, каким является (27) при определенном выборе знака коэффициентов. Важно отметить, что любая суперпозиция $v_{N}$ простых решений $\Psi_{i}$ удовлетворяет данному уравнению. Это дает альтернативный способ нахождения решений таких уравнений с помощью рядов или произведений.

\section{6. ПРЕОБРАЗОВАНИЕ К СТАНДАРТНОЙ ФОРМЕ}

Рассмотрим теперь вопрос о применимости суперпозиционных решений $u_{N}$ для вычисления решений исходного уравнения нелинейной диффузии (3). Рассмотрим функции

$$
\begin{gathered}
S(x, t)=a(t)\left(P_{1}\left(\Phi_{1}, \ldots, \Phi_{N}\right)+b(x, t) P_{0}\left(\Phi_{1}, \ldots, \Phi_{N}\right)\right)=a(t) \frac{A^{N}}{Z^{N}} \sigma(Z, t), \\
\sigma(Z, t)=\frac{Z}{A} P_{1}(\mathbf{Z})+b(x, t) P_{0}(\mathbf{Z}), \quad F(x, t)=\frac{\partial^{2}}{\partial x^{2}} \ln S .
\end{gathered}
$$

Функция $a(t)$ может быть любой дифференцируемой функцией времени, а функцию $b(x, t)$ мы выберем далее. Используя тождества (18) и (19), находим

$$
F(x, t)=\frac{\partial^{2}}{\partial x^{2}} \ln S(x, t)=k^{2} Z \frac{\partial}{Z}\left(Z \frac{\partial}{\partial Z} \ln \sigma(Z, t)\right) .
$$

Имеют место два тождества, одно из которых получается следующим образом:

$$
\begin{aligned}
\frac{\partial}{\partial x} u_{N} & =-\frac{1}{A k} \frac{\partial^{2}}{\partial x^{2}} \ln \frac{P_{0}(\mathbf{Z})}{a(t) Z P_{1}(\mathbf{Z}) A^{-1}(t)+a(t) b(x, t) P_{0}(\mathbf{Z})}-\frac{F(x, t)}{A k}= \\
& =\frac{1}{A k} \frac{\partial^{2}}{\partial x^{2}} \ln \left(a(t) \frac{Z}{A} \frac{P_{1}(\mathbf{Z})}{P_{0}(\mathbf{Z})}+a(t) b(x, t)\right)-\frac{F(x, t)}{A k}= \\
& =\frac{1}{A k} \frac{\partial^{2}}{\partial x^{2}} \ln w_{N}-\frac{F(x, t)}{A k},
\end{aligned}
$$

где $w_{N}=a\left(u_{N}+b\right)$. Второе тождество

$$
u_{N}=\frac{1}{A k} \frac{\partial}{\partial x} \ln w_{N}-\frac{J(x, t)}{A k}+\frac{N}{A}
$$

получается аналогичным способом. Здесь

$$
J(x, t)=\frac{\partial \ln S}{\partial x}=\frac{\partial}{\partial x} \ln \sigma(Z, t)-N k .
$$


Тождество (31) можно, используя (30), переписать так:

$$
\begin{aligned}
a \frac{\partial u_{N}}{\partial x} & =\frac{a}{A k} \frac{\partial^{2}}{\partial x^{2}} \ln w_{N}-\frac{a F}{A k}= \\
& =A k\left(u_{N}+\frac{J(x, t)}{A k}-\frac{N}{A}\right) w_{N}-\frac{\partial a}{\partial x} u_{N}-a \frac{\partial b}{\partial x} .
\end{aligned}
$$

Теперь, используя только тождество (30), приведем уравнение (22) к нужной форме (2) без квадратичной нелинейности:

$$
\frac{\partial u_{N}}{\partial t}-\left(\mathcal{D}_{0}-\frac{\gamma}{A^{2} k^{2}}\right) \frac{\partial^{2}}{\partial x^{2}} \ln w_{N}+\left(\mu+\frac{\gamma}{A}\right) u_{N}+\left(\mathcal{D}_{0}-\frac{\gamma}{A^{2} k^{2}}\right) F=0
$$

используя еще и тождество (32), это уравнение можно привести к более общему виду

$$
\begin{aligned}
\frac{\partial u_{N}}{\partial t} & -\left(\mathcal{D}_{0}-\frac{\gamma}{A^{2} k^{2}}-\frac{G a}{A k}\right) \frac{\partial^{2}}{\partial x^{2}} \ln w_{N}+\left(\mu+\frac{\gamma}{A}\right) u_{N}- \\
& -G\left[A k\left(u_{N}+\frac{J(x, t)}{A k}-\frac{N}{A}\right) w_{N}-\frac{\partial a}{\partial x} u_{N}\right]+ \\
& +G a \frac{\partial b}{\partial x}+\left(\mathcal{D}_{0}-\frac{\gamma}{A^{2} k^{2}}-\frac{G a}{A k}\right) F=0
\end{aligned}
$$

где $G=G(x, t)$ - произвольная функция координат и времени.

Предполагая, что функция $a=a(t)$ является только функцией времени, в уравнении (33) удобно сделать подстановку $u_{N}=\left(w_{N}-b\right) / a$ и ввести новую переменную времени $\tau$ такую, что $d \tau=a(t) \mathcal{D} d t$. Здесь

$$
\mathcal{D}=\mathcal{D}_{0}-\frac{\gamma}{A^{2} k^{2}}
$$

В этом случае уравнение (33) приобретет вид уравнения (3):

$$
\frac{\partial w_{N}}{\partial \tau}-\frac{\partial^{2}}{\partial x^{2}} \ln w_{N}+m(t) w_{N}+g=0
$$

где

$$
\begin{gathered}
m(t)=\frac{a(A \mu+\gamma)-A \dot{a}}{a^{2} \mathcal{D} A}, \quad g(x, t)=-\frac{1}{\mathcal{D}} Q(x, t)+F(x, t), \\
Q(x, t)=\frac{\partial b}{\partial t}+\left(\mu+\frac{\gamma}{A}\right) b .
\end{gathered}
$$

Аналогично преобразуется уравнение (34) с помощью подстановки $u_{N}=w_{N} / a-b$ и новой переменной $\tau$ такой, что $d \tau=a(t) \mathcal{D}_{G}(t) d t$, где

$$
\mathcal{D}_{G}=\mathcal{D}_{0}-\frac{\gamma}{A^{2} k^{2}}-\frac{G a}{A k}
$$

Полагая $G=G(t)$ и $a=a(t)$, имеем

$$
\frac{\partial w_{N}}{\partial \tau}-\frac{\partial^{2}}{\partial x^{2}} \ln w_{N}+m_{1}(x, t) w_{N}+\gamma_{1}(t) w_{N}^{2}+g(x, t)=0 .
$$


Здесь

$$
\begin{gathered}
m_{1}(t, x)=\frac{1}{a^{2} \mathcal{D}_{G}}\left[-\frac{d a}{d t}+\left(\mu+\frac{\gamma}{A}\right) a-G a^{2} k N-G Y(x, t)\right], \\
\gamma_{1}(t)=-\frac{G A k}{a \mathcal{D}_{G}}, \quad g(x, t)=-\frac{1}{\mathcal{D}_{G}}\left(Q(x, t)-\mathcal{D}_{G} F(x, t)-G(t) \frac{\partial b}{\partial x}\right), \\
Y(x, t)=A k\left(b(x, t) a-a^{2} \frac{J(x, t)}{A k}\right) .
\end{gathered}
$$

Таким образом, в случае $a=a(t)$ и $G=G(t)$ при произвольных функциях времени $\mu(t), \gamma(t), \mathcal{D}_{0}(t)$ и произвольной функции $b(x, t)$ функция $w_{N}=a\left(u_{N}+b\right)$, где $u_{N}$ определено в (21), удовлетворяет уравнениям нелинейной диффузии (33) и (34).

\section{7. УСЛОВИЯ КОМПЛЕМЕНТАРНОСТИ}

ОПРЕДЕЛЕНИЕ 1. Под условием комплементарности совокупности решений $\Phi_{i}$, $i=1, \ldots, N$, уравнения (4) будем понимать такой выбор коэффициентов этих функций, а также вспомогательных функций $a(t)$ и $b(Z, t)$, фигурирующих в определении функций $F(x, t)$ и $S(x, t)$, при которых уравнения (35) или (37) сводятся к исходному уравнению (3) с коэффициентами, являющимися функциями заданного вида, которые зависят только от переменной $t$.

Чтобы найти условия комплементарности, рассмотрим в качестве $b(x, t)$ функцию

$$
b(x, t)=\beta(Z) A^{-1}(t)
$$

где $\beta(Z)$ - аналитическая по $Z$ функция. В результате функция $\sigma(Z, t)$, заданная в (28), примет следующий вид:

$$
\sigma(Z, t)=\frac{1}{A(t)} \xi(Z), \quad \xi(Z)=Z P_{0}^{\prime}(Z)+\beta(Z) P_{0}(Z) .
$$

Используя эти соотношения, вычислим функцию $g(x, t)$ в уравнении (35). Согласно (29) имеем

$$
F(x, t)=\frac{\partial^{2}}{\partial x^{2}} \ln S=k^{2} Z \frac{\partial}{\partial Z}\left(Z \frac{\partial \ln \xi(Z)}{\partial Z}\right) .
$$

Далее,

$$
Q(x, t)=\left[\frac{\partial}{\partial t} \frac{1}{A}+\left(\mu+\frac{\gamma}{A}\right) \frac{1}{A}\right] \beta(Z)+\frac{1}{A} \frac{\partial \beta(Z)}{\partial t}=\frac{R}{A} Z \frac{\partial \beta}{\partial Z} .
$$

Здесь $R(t)=\dot{A} / A-\dot{\Theta}$ и было использовано уравнение (7) для $A$. Подставляя полученные соотношения для $F(x, t)$ и $Q(x, t)$ в выражение $(36)$ для функции $g(x, t)$, находим

$$
g(x, t)=-\frac{R}{\mathcal{D} A} Z \frac{\partial \beta}{\partial Z}+k^{2} Z \frac{\partial}{\partial Z}\left(Z \frac{\partial \ln \xi(Z)}{\partial Z}\right) .
$$

Полагая

$$
\frac{R}{A \mathcal{D}}=\lambda
$$


выводим общее уравнение, связывающее функции $\beta(Z)$ и $P_{0}(\mathbf{Z})$, при котором функция $g(x, t)$ является некоторой вещественной постоянной $g_{0}$ :

$$
g_{0}=-\lambda Z \frac{\partial \beta}{\partial Z}+k^{2} Z \frac{\partial}{\partial Z}\left(Z \frac{\partial \ln \xi(Z)}{\partial Z}\right) .
$$

Интегрируя это уравнение один раз по $Z$, приходим к уравнению

$$
g_{1}+g_{0} \ln Z=-\lambda \beta(Z)+k^{2} Z \frac{\partial \ln \xi(Z)}{\partial Z},
$$

которое и представляет собой общее условие комплементарности функций $\Phi_{i}$, входящих в суперпозицию $u_{N}$, определенную соотношениями (21). В (42) $g_{1}$ - постоянная интегрирования.

Результат можно сформулировать в виде утверждения.

УТВЕРЖДЕНИЕ 3. Если выполнены условия (40) и (42), то функиия

$$
w_{\beta}(x, t)=-\frac{a(t)}{A(t)}\left[\frac{1}{k}\left(\frac{\partial}{\partial x} \ln P_{0}(Z(x, t))\right)+\beta(Z(x, t))\right]
$$

есть решение уравнения (35) при прочзвольных постоянных $k, \lambda, g_{1}, g_{0}$ с коэфбиииентом $m=m(t)$ и $g \equiv g_{0}$.

Для завершения исследования рассмотрим решения уравнения (42). Введем для удобства функцию $\zeta(Z)=\beta(Z)+P_{0}^{\prime} / P_{0}$ и переменную $y=\ln Z$. В этом случае уравнение (42) примет вид

$$
\frac{d \zeta}{d y}-\lambda \zeta^{2}+U(y) \zeta=0
$$

где

$$
U(y)=\left(k^{2}+\lambda\right) \frac{d \ln P_{0}}{d y}-g_{1}-g_{0} y .
$$

Это уравнение Риккати легко интегрируется, в результате чего получаем выражение для функции $\zeta$ :

$$
\zeta(Z)=-\frac{Z}{\lambda} \frac{d}{d Z} \ln \eta(\ln Z)
$$

где

$$
\eta(y)=\eta_{1} \int^{y} \exp \left(-\int^{y} U\left(y^{\prime \prime}\right) d y^{\prime \prime}\right) d y^{\prime}+\eta_{0}=\eta_{1} \int^{y} \frac{e^{-g_{1} y^{\prime}-g_{0} y^{\prime 2}} d y^{\prime}}{P_{0}^{k^{2}+\lambda}\left(e^{y^{\prime}}\right)}+\eta_{0},
$$

а $\eta_{0}, \eta_{1}-$ постоянные интегрирования. Соответственно

$$
w_{\beta}(x, t)=-\frac{a}{A(t)} \frac{1}{k} \frac{\partial}{\partial x} \ln \zeta(Z(x, t))=\frac{a}{A(t)} \frac{1}{\lambda k^{2}} \frac{\partial^{2}}{\partial x^{2}} \ln \eta(\ln Z(x, t)) .
$$

Выбор порядка $N$ и коэффициентов полинома $P_{0}(Z)$ произволен, что также эквивалентно произвольности выбора чисел $z_{i}=-B_{i}$, поэтому полученные соотношения позволяют решать задачу Коши на всей оси координат для начальных распределений концентрации, заданных как аналитические функции координаты $x$. Это 
связано с тем, что, переходя к пределу $N \rightarrow \infty$, вместо полинома $P_{0}(Z)$ мы можем брать любую аналитическую в нуле по $Z$ функцию. Поскольку в начальный момент времени переменная $Z$ является аналитической функцией от $x$, соответствующие решения могут быть согласованы с почти любым гладким начальным распределением концентрации. Сложности могут возникать в случае, когда строится решение задачи с $g_{0} \neq 0$. В этом случае в выражении для $F(\ln Z)$ явно содержится слагаемое, пропорциональное $\ln Z$, которое неаналитично в нуле. Однако анализ данной ситуации полезно проводить при решении конкретных задач.

Важными являются случаи, когда в (44) можно вычислить интеграл в правой части в аналитическом виде. Он вычисляется достаточно просто, если выполнены следующие условия: $g_{0}=0, g_{1}=L, k^{2}+\lambda=M$, где $M$ и $L$ - целые числа, а порядок $N$ полинома $P_{0}(Z)$ конечен. В частности, при $M<0$ и $g_{0}=0$ для любого конечного $N$ интеграл в (44) является полиномом по $Z$.

\section{8. РЕШЕНИЕ УРАВНЕНИЯ С КВАДРАТИЧНОЙ НЕЛИНЕЙНОСТЬЮ}

Аналогичные вычисления можно проделать и для уравнения (37). При сделанном выборе функции $b(x, t)$ в форме (39) вычисление функции $g(x, t)$, определенной соотношениями (38), дает

$$
g(x, t)=-\frac{R+k G}{\mathcal{D}_{G} A} Z \frac{\partial \beta}{\partial Z}+k^{2} Z \frac{\partial}{\partial Z}\left(Z \frac{\partial \ln \xi(Z)}{\partial Z}\right) .
$$

Отсюда вытекает, что эта функция будет постоянной, если выполнено условие

$$
\frac{R+k G}{\mathcal{D}_{G} A}=\lambda=\text { const. }
$$

При этом функции $\beta(Z)$ и $P_{0}(\mathbf{Z})$ связаны тем же уравнением $(41)$ и, как следствие, уравнением (42).

Вычислим теперь функцию $Y(x, t)$, входящую в коэффициент $m_{1}(x, t)$ уравнения (37). Подставляя в нее выражения для $b$ и $J$, с учетом условия комплементарности находим

$$
Y(x, t)=a^{2} k\left(\beta(Z)+Z \frac{\partial \ln \xi(Z)}{\partial Z}-N\right) .
$$

Чтобы эта функция зависела только от переменной $t$, необходимо и достаточно, чтобы выполнялось еще одно условие:

$$
\beta(Z)+Z \frac{\partial \ln \xi(Z)}{\partial Z}=\beta_{0}=\text { const }
$$

где $\beta_{0}$ - произвольная вещественная постоянная. При этом коэффициент $m_{1}(t)$ в уравнении (37) будет зависеть только от $t$ и иметь вид

$$
m_{1}(t)=\frac{1}{a^{2} \mathcal{D}_{G}}\left[-\frac{d a}{d t}+\left(\mu+\frac{\gamma}{A}\right) a-G a^{2} \beta_{0}\right] .
$$


Следовательно, доказано

УтвеРЖДЕНИЕ 4. Функиия $w_{\beta}(x, t)$, определенная соотношением (43) в утверждении 3, при произвольных постоянных $\lambda, \beta_{0}, k$ является решением уравнений (37) с коэфбициентами $m_{1}=m_{1}(t), \gamma_{1}=\gamma_{1}(t)$, зависящими только от $t$, u $g(t) \equiv g_{0}$, если функции $\beta(Z)$ и $P_{0}(\mathbf{Z})$ свлзаны уравнениями (42) и (45) и выполнено условие

$$
\frac{R+k G}{\mathcal{D}_{G} A}=\lambda .
$$

Вычисление функциональной формы решений в этом случае приводит при произвольных постоянных $g_{0}$ и $\beta_{0}$ к определению явного вида и $\beta(Z)$, и $P_{0}(Z)$ :

$$
\beta(Z)=\beta_{0}-Z \frac{d \ln \xi(Z)}{d Z}, \quad P_{0}(Z)=Z^{-\beta_{0}} \xi(Z)\left(q_{0}+\frac{Z}{\beta_{0}+1}\right),
$$

где

$$
\xi(Z)=e^{W(Z)}, \quad W(Z)=Z^{-\lambda / k^{2}}\left(c_{1}+\int^{Z} Z^{\prime \lambda / k^{2}}\left(\left(g_{0}+\lambda \beta_{0}\right) \ln Z^{\prime}+g_{1}\right) d Z^{\prime}\right) .
$$

Функция $W(Z)$ вычисляется в явном виде при любых значениях параметров решения. В частности,

$$
W(Z)=Z^{-\lambda / k^{2}} c_{1}+\frac{Z}{\lambda / k^{2}+1}\left(\left(g_{0}+\lambda \beta_{0}\right) \ln Z+g_{1}\right)-\frac{\left(g_{0}+\lambda \beta_{0}\right)}{\left(\lambda / k^{2}+1\right)^{2}} Z .
$$

Эти решения уже не содержат произвольных функциональных параметров и описывают ограниченный класс точных решений уравнения (3). Все замечания о решениях, построенных в предыдущем разделе, остаются справедливыми и для решений, построенных в данном разделе.

\section{9. ЗАКЛЮЧЕНИЕ}

Развитый в предложенной работе метод представления суперпозиции простых решений производными от полиномов позволил построить решение задачи Коши для уравнения нелинейной диффузии с нелинейным источником в форме линейной или квадратичной функции концентрации. Поскольку решения строятся практически для любого начального распределения, в рамках такого подхода можно решать задачи о формировании регулярных и периодических структур в среде с нелинейной диффузией. В работе из-за ограниченности объема статьи не проводились вычисления для решений $\Phi$ полиномиального по $x$ вида. Для таких решений уравнения для суперпозиционных функций отличаются от тех, которые были получены для решений с экспонентами. Однако все необходимые построения можно воспроизвести по аналогии с приведенными в статье. Более того, развитый метод построения суперпозиционных решений полиномиального типа может быть полезным для более широкого круга нелинейных задач. В частности, связь между суперпозициями решений некоторых нелинейных уравнений с решениями линейных уравнений может рассматриваться в качестве одного из способов строить решения таких уравнений с переменными коэффициентами заданного типа. Это было продемонстрировано на примере суперпозиций полиномов по переменной $T$ как решений телеграфного уравнения. 
Благодарности. Автор благодарит за полезные обсуждения результатов данной работы И. О. Золотовского и Д. А. Коробко. Работа выполнена при поддержке Министерства образования и науки РФ (в рамках Государственного задания и проекта № 14.Z50.31.0015), а также при частичной финансовой поддержке РФФИ в рамках проекта 13-01-97067 р-поволжье_а.

\section{Список литературы}

[1] А. А. Самарский, А.П. Михайлов, Математическое моделирование. Идеи. Методъ. Примеры, Наука, М., 1997.

[2] А.А. Самарский, В.А. Галактионов, С.П. Курдюмов, А.П. Михайлов, Режимъ с обострением в задачах для квазилинейных параболических уравнений, Наука, М., 1987.

[3] В. В. Пухначев, Прикл. механика и техн. физика, 36:2 (1995), 23-31.

[4] С. Н. Аристов, Прикл. механика и техн. физика, 40:1 (1999), 22-26.

[5] В. М. Журавлев, ТМФ, 124:2 (2000), 265-278.

[6] В. М. Журавлев, Писъма в ЖЭТФ, 75:1 (2002), 11-16.

[7] В. М. Журавлев, ЖЭТФ, 129:3 (2006), 587-604.

Поступила в редакцию 19.08.2014, после доработки 8.10.2014 